\title{
Validez y Confiabilidad del Índice de Satisfacción Vital (LSI-A) de Neugarten, Havighurst \& Tobin en una Muestra de Adultos y Adultos Mayores en Chile ${ }^{1}$
}

\section{Validity and Reliability of the Neugarten, Havighurst \& Tobin's Life Satisfaction Index (LSI-A), in a Sample of Adults and Older Adults in Chile}

\author{
Beatriz Zegers Prado \\ Cristian Rojas-Barahona \\ Universidad de los Andes, Chile \\ Carla Förster Marín \\ Pontificia Universidad Católica de Chile
}

(Rec: 11 septiembre $2008 \quad$ Acep: 10 mayo 2009)

\begin{abstract}
Resumen
Se presentan los resultados obtenidos en la estimación de la confiabilidad y la validez del Índice de Satisfacción Vital (LSI-A) de Neugarten, Havighurst \& Tobin $(1961,1996)$. La muestra estuvo constituida por 473 adultos de 30 a 75 años (hombres y mujeres) de la Región Metropolitana de Santiago de Chile, distribuidos en tres niveles de ingreso, tres grupos de edad y tres niveles de escolaridad. Se estimó la validez de contenido, concurrente y de constructo del instrumento, definiendo 5 factores que explican el $50.62 \%$ de la varianza. La confiabilidad con alpha de Cronbach fue de 0.80 para la muestra completa, el índice varió entre 0.77 a 0.83 según edad, entre 0.77 y 0.81 según nivel educacional y entre 0.74 y 0.83 según nivel de ingreso; no se observaron variaciones según sexo $(0.80)$.

Palabras Claves: Satisfacción vital, adulto mayor, bienestar subjetivo, validación.
\end{abstract}

\begin{abstract}
The findings of a research aimed to estimate reliability and validity of The Life Satisfaction Index (LSI-A) of Neugarten, Havighurst \& Tobin $(1961 ; 1996)$ are presented. The sample consisted of 473 adults from 30 to 75 years (male and female) from Metropolitan Region of Santiago, Chile, distributed into three levels of income, age groups and educational levels. The content, concurrent and construct validity of the instrument was estimated, defining five factors accounting for $50.62 \%$ of the variance. The reliability with Cronbach's alpha was 0.80 for the full sample, the index ranged between 0.77 to 0.83 depending on age, between 0.77 and 0.81 according to educational level and between 0.74 and 0.83 depending on income level, no changes were observed by sex $(0.80)$.
\end{abstract}

Key Words: Life-satisfaction, older-adult, subjective welfare, validation.

Correspondencia a: bzegers@uandes.cl

Este trabajo ha sido elaborado como parte del Proyecto de Investigación PSI 001/2007, financiado con el Fondo de Ayuda a la Investigación de la Universidad de los Andes, Santiago de Chile. 


\section{Introducción}

El Índice de Satisfacción Vital-A (LSI-A) se desarrolló en la década de los sesenta del siglo pasado por Neugarten, Havighurst \& Tobin (1961). Específicamente, realizaron una investigación cuyo objetivo fue definir una medida operacional válida y confiable del bienestar psicólogico entre adultos mayores. Su brevedad, fácil administración, medidas de validez y confiabilidad preliminares apropiadas, han hecho de él un instrumento muy atractivo, traduciéndose en una variedad de investigaciones y aplicaciones en distintos países y contextos (por ejemplo, Chiang, Lu, Chu, Chang \& Chou, 2008; Sener, Terzioglu \& Karabulut, 2007). Dado que el concepto de calidad de vida está en estrecha relación con el bienestar subjetivo y con una amplia variedad de circunstancias vitales, se trata de un Índice cuyas aplicaciones trascienden el área de la investigación en gerontología (Inga y Vara, 2006; Lobello, Underhill \& Fine, 2004), pudiendo también ser útil cuando se quiere evaluar el éxito de la adaptación o felicidad en cualquier momento de la vida adulta. De allí que el objetivo de este trabajo sea examinar la consistencia interna del LSI-A de Neugarten y colaboradores $(1961 ; 1996)$, la validez concurrente entre la LSI-A y la Escala de Autoestima de Rosenberg (1965) y su validez de constructo a través del procedimiento de análisis factorial de componentes principales con rotación Varimax, en una población de adultos y adultos mayores chilenos. Este último análisis no fue realizado por Neugarten y colaboradores $(1961 ; 1996)$, aunque concluyeron que a partir de las correlaciones encontradas entre las dimensiones, existe cierto grado de independencia entre ellas, apoyando el supuesto que el Índice estaba compuesto por más de una dimensión o factor, asunto que es también objeto de este trabajo.

La satisfacción vital es un constructo psicológico que refleja el bienestar subjetivo del individuo. Su estudio fue iniciado por Neugarten y colaboradores $(1961 ; 1996)$ en la década de los sesenta del siglo pasado. Los investigadores distinguieron inicialmente dos formas de aproximarse a su medición: las primeras, centradas en las conductas manifiestas y cuya evaluación se basa en criterios sociales de éxito o competencia, número de actividades y grado de participación social; las segundas, consideran la apreciación subjetiva y prestan secundaria atención a factores sociales, privilegiando la estimación del propio sujeto respecto de su vida actual y pasada, su felicidad y satisfacción. Es esta última perspectiva la adoptada por Neugarten y colaboradores $(1961$; 1996) cuando definieron operacionalmente el constructo, señalando que a la base del mismo existían cinco dimensiones: a) Entusiasmo (entusiasmo y compromiso en las actividades, personas e ideas con las cuales el sujeto se involucra; se opone la indiferencia, abatimiento y apatía); b) Resolución y Fortaleza (responsabilidad por la propia vida, se contrapone la resignación o la aceptación pasiva de lo que la vida le ofrece), c) Congruencia entre objetivos deseados y alcanzados (logro y éxito en las metas propuestas en la vida, cualesquiera ellas hayan sido; lo contrario se encuentra cuando el individuo siente que ha perdido la mayoría de las oportunidades), d) Autoconcepto positivo (personas que se preocupan por su cuidado y apariencia, y que se evalúa como alguien sabio y maduro; lo opuesto se encuentra en aquellos que se sienten viejos, débiles, enfermos, incompetentes) y e) Tono Emocional (presencia de actitudes optimistas, felices, sano humor y tono afectivo positivo hacia los demás; se ubicarían en el otro extremo aquellos que son pesimistas, quejosos, se sienten solos o se enojan cuando están con otros).

El énfasis en la importancia de la visión subjetiva al evaluar la vida y el reconocimiento de que el bienestar incluye elementos positivos que trascienden la prosperidad económica, es plenamente aceptado hoy día, aunque se reconoce la complejidad implícita en su evaluación, considerándose "una amplia categoría de fenómenos que incluyen las respuestas emocionales, el dominio de satisfacciones y el juicio global de la satisfacción vital" (Diener, Suh, Lucas \& Smith, 1999, p. 277). Con todo, las investigaciones en este campo se han desarrollado en gran parte siguiendo caminos paralelos: los juicios cognitivos sobre satisfacción de vida y las emociones (Diener \& Lucas, 1999, en Inga \& Vara, 2006). Para Neugarten y colaboradores (1961; 1996), la satisfacción vital incluye ambos dominios, allí radica precisamente una de las razones por las cuales se ha optado por la conceptualización entregada por estos investigadores y que se encuentra plenamente vigente.

Dado que el concepto de calidad de vida está en estrecha relación con el bienestar subjetivo, es que se trata de una medida que puede ser de utilidad cuando se quiere evaluar el éxito de la adaptación o felicidad en cualquier momento de la vida adulta (Olson-Madden, 2008). En este sentido, Smith, Borchelt, Maier y Jopp (2002) consideran relevante incorporar medidas de salud subjetiva o de bienestar como la satisfacción vital en estudios sobre el envejecimiento, ya que la mayoría se han centrado en la depresión y la demencia.

La complejidad del constructo de la satisfacción vital se refleja en múltiples estudios, como por ejemplo el realizado por Staudinger, Fleeson y Baltes (1999). En el citado estudio se observan bajas correlaciones entre condiciones objetivas de la calidad de vida y el bienestar subjetivo en adultos de todas las edades, fenómeno que se conoce como "la paradoja del bienestar". También este constructo se encuentra afectado por factores externos, como: salud, ingresos, nivel de educación, creencias religiosas, estado civil, entre otros; así como por factores personales internos: temperamento y cogniciones, metas, esfuerzos por moderar las influencias de las circunstancias vitales en la adaptación del individuo (Diener, Suh, Lucas \& Smith, 1999). 
Tabla 1. Correlaciones entre dimensiones del LSR

\begin{tabular}{lcccc}
\hline & Resolución & Congruencia & Autoconcepto & Tono Emocional \\
\hline Entusiasmo & 0.67 & 0.56 & 0.79 & 0.84 \\
Resolución & & 0.70 & 0.83 & 0.48 \\
Congruencia & & & 0.75 & 0.57 \\
Autoconcepto & & & & 0.82
\end{tabular}

\section{La Escala de Satisfacción Vital (LSI-A) de Neugarten, Havighurst \& Tobin $(1961,1996)$}

La construcción del LSI-A se inició como parte del estudio de campo "Ciudad de Kansas sobre la vida Adulta" (Kansas City Study of Adult Life), orientado a definir una medida operacional, válida y confiable del bienestar psicológico entre adultos mayores. La muestra inicial estuvo conformada por 177 personas distribuidas en tres niveles socio-económicos y cinco grupos de edad al momento de la primera entrevista (50-56 años hasta 80-89 años). La población provenía de dos grupos: el primero conformado por personas cuyas edades fluctuaban entre 50 y 70 años, residentes del área metropolitana de la ciudad de Kansas, estratificada probabilísticamente según nivel socioeconómico, excluyéndose enfermos crónicos o discapacitados físicamente. El segundo se incorporó dos años después; incluyó personas entre 70 y 90 años y se usó un muestreo por cuoteo, se excluyeron a aquellos deprivados económicamente y demenciados. Los datos se recolectaron a través de 4 rondas de entrevistas realizadas a cada individuo. Se les preguntó por patrones de la vida diaria y fines de semana, actitudes y valores, relaciones sociales, ingresos y trabajo, voluntariado en organizaciones religiosas, actitudes hacia el envejecimiento, enfermedad, muerte, inmortalidad, soledad, aburrimiento, rabias, modelos de roles y auto-imagen. Las entrevistas para el segundo grupo combinaron preguntas estructuradas y otras extraídas de las primeras tres entrevistas realizadas al primer grupo.

En un segundo momento, los investigadores denominaron al constructo "Satisfacción Vital" y definieron operacionalmente cinco dimensiones: 1) Entusiasmo 2) Resolución y fortaleza, 3) Congruencia entre objetivos deseados y alcanzados, 4) Autoconcepto positivo y 5) Tono emocional. Cada dimensión se calificó en una escala de cinco puntos (a mayor puntaje mayor valoración positiva de la dimensión), labor que fue realizada por jueces que no conocían ni habían entrevistado a los sujetos participantes, obteniéndose como resultado el "Life Satisfaction Ratings" (LSR).

Los individuos fueron ubicados en el polo positivo del continuo de Satisfacción Vital cuando: a) consideraban placenteras las actividades de la vida diaria, b) evaluaban sus vidas como significativas y aceptaban las decisiones que habían tomado a lo largo de ella, c) sentían que habían al- canzado los logros planteados, d) tenían una imagen positiva de sí mismos y e) el humor era optimista y feliz.

Para los 177 casos, los puntajes del LSR variaron de 8 a 25 puntos (con un mínimo de 1 y un máximo de 25 puntos), con un promedio de 17.8 y una D.S. de 4.6.

Para estimar la confiabilidad del LSR se consideraron las codificaciones realizadas por dos jueces independientes, participando un total de 14 jueces. Se obtuvo un coeficiente inicial de consistencia interna de 0.78 . Teniendo en cuenta que en las subsiguientes etapas se usó el promedio del puntaje de los dos evaluadores, se empleó el coeficiente de atenuación de Spearman Brown, con el que éste aumentó a 0.87 . El acuerdo interjueces para la escala total o en una de las dimensiones fue de 94\%, 97\% para Entusiasmo, 96\% para Resolución, 92\% en Congruencia, 96\% para Autoconcepto y $92 \%$ en Tono Emocional.

Los resultados de las correlaciones entre las dimensiones se muestran en la Tabla 1. Se aprecian correlaciones positivas entre ellas, aunque también se observa un cierto grado de independencia, apoyando el supuesto de que la escala está compuesta por más de una dimensión. Estos coeficientes no fueron sometidos a un análisis factorial, pero sus autores señalaron que las dimensiones Entusiasmo, Tono Emocional y posiblemente, Autoconcepto, constituían un solo factor y que probablemente existiera uno o dos factores que estuvieran operando en la matriz (Neugarten et al., 1961; 1996).

No se encontraron correlaciones significativas entre el LSR y edad, entre LSR y sexo. Tampoco entre LSR y el Índice de Características Socio Económicas basado en área de residencia, nivel de educación y ocupación.

Para validar el LSR los investigadores buscaron el grado de acuerdo entre la codificación de los jueces que leyeron el material de las exploraciones y que no conocían a los sujetos, y la puntuación de una nueva entrevista realizada por clínicos expertos a 80 sujetos de los 177 de la muestra inicial (algunos se habían cambiado, otros habían fallecidos, los terceros se rehusaron a continuar participando). Los clínicos no tenían información acerca de los individuos, ni tampoco discutieron los casos con otros miembros del equipo. Establecieron un nuevo puntaje: LSR-C1, este promedio de puntaje fue ligeramente superior al obtenido para los 177 (18.9 vs. 17.8), aunque estos 80 individuos eran representativos de la muestra inicial en cuanto a sexo, edad y clase social. 
La correlación obtenida entre el puntaje promedio otorgado por los dos jueces que calificaron la entrevista individual y el LSR-C1 para los 80 casos, fue de 0.64. El porcentaje de acuerdo para la dimensión Entusiasmo fue de $86 \%, 76 \%$ para Resolución, $73 \%$ en Congruencia, 78\% para Autoconcepto y $69 \%$ en Tono Emocional. Esta correlación fue considerada satisfactoria teniendo en cuenta: a) el tiempo transcurrido entre las primeras cuatro entrevista y la quinta (varios sujetos habían sufrido cambios significativos en sus condiciones de vida), b) un porcentaje de las personas que habían alcanzados bajos puntajes en el LSR abandonaron el estudio lo que disminuyó la variabilidad de puntajes, c) que el LSR se basó en datos de entrevistas grabadas y el LSR-C1 se extrajo de entrevistas cara a cara, y d) la mayor profundidad que tuvieron las entrevistas clínicas.

Los autores destacan que la correlación entre el LSR y el LSR-C1 fue más alta para los individuos de mayor edad de la muestra. Para los 30 casos de 70 o más años, la correlación fue de 0.70 ; mientras que para los restantes (menores de 69 años), fue de 0.53. Los investigadores explican que estas diferencias podrían deberse a que los mayores posiblemente mostraron una menor tendencia a entregar respuestas que se conformaran a expectativas sociales que aquellos de menor edad y, en segundo lugar, podría ser que las preguntas desarrolladas para conformar el LSR fueran más apropiadas para los sujetos de mayor edad, aunque ello no se debió a algo conciente de parte de ellos.

No obstante la riqueza de la información proporcionada por el LSR, se trata de un sistema que requiere de una gran cantidad de tiempo para su administración y tabulación. Por este motivo, los investigadores, usando el LSR como referencia, confeccionaron dos instrumentos de autoreporte, cuya administración tomara pocos minutos y que pudiesen administrarse conjunta o separadamente. Para ello se seleccionaron 60 sujetos que habían obtenido tanto altos como bajos puntajes en el LSR y que representaran la muestra inicial en cuanto a edad, sexo y clase social. De sus entrevistas escogieron términos y preguntas abiertas y cerradas, a fin de diferenciar entre los grupos extremos, y agregaron nuevos ítemes, buscando reflejar las cinco dimensiones del LSR. Surgieron dos instrumentos preliminares: el "Life Satisfaction Index A" (LSI-A), con 25 ítemes que se respondían de acuerdo o en desacuerdo, y el "Life Satisfaction Index B" (LSI-B), con 17 preguntas abiertas y un "chenk list", puntuadas en una escala de tres puntos ( 0,1 ó 2 puntos).

Luego se aplicaron los dos instrumentos a 92 individuos, realizándose los cálculos con sólo 60 de ellos. La correlación entre el puntaje del LSR y el LSI-A fue de 0.52 , mientras que con el LSI-B fue de 0.59. Una vez completada la administración a los 92 individuos, se procedió a realizar un análisis de ítemes a fin de evaluar el grado de discriminación entre altos y bajos puntajes, descartándose cinco ítems del LSI-A y siete del LSI-B.
Finalmente, la correlación entre LSI-A y LSR fue de 0.55 , con un promedio de 12.4 y una D.S. 4.4 y para el LSI-B y LSR fue 0.58 , con un promedio de 15.1 y D.S. de 4.7. Los puntajes combinados de ambas escalas correlacionaron con el LSR en 0.61, el promedio para los puntajes combinados fue 27.6 y D.S. 6.7.

A continuación se detalla el proceso de validación realizado.

\section{Método}

\section{Participantes}

La muestra estuvo conformada por 473 adultos de la Región Metropolitana de Santiago, Chile, en un muestreo intencionado por cuotas, distribuidos en tres niveles de ingreso: 151 en el nivel bajo (0-84 mil pesos), 172 en el medio (85-264 mil pesos) y 150 en el alto (265 mil pesos o más) de acuerdo con la clasificación de ingreso autónomo per cápita del hogar presentado por MIDEPLAN en la encuesta CASEN 2003 para la Región Metropolitana urbana; cabe señalar que el nivel bajo considera los quintiles I y II, el nivel medio los quintiles III y IV, y el nivel alto el quintil V. El número de hombres y de mujeres es similar en cada nivel y se consideraron tres grupos de edad: $30-45$ años, 45-59 años y 60-75 años. Esta estratificación tuvo en cuenta que la Adultez Mayor comienza, de acuerdo a la definición dada por Naciones Unidas, a los 60 años y los estudios de Levinson (1978; 1996), que sitúan el comienzo de la Adultez Media a los 45 años; si bien la Adultez Joven se iniciaría en promedio a los 28 años de acuerdo a este mismo autor, se mantuvo el rango de 15 años para delimitar los otros dos grupos etáreos. El nivel de escolaridad fue categorizado en tres grupos: básica, media y superior (técnica-universitaria). Hay que señalar que fue difícil encontrar sujetos con niveles de escolaridad baja en el grupo de menor edad, debido posiblemente al aumento en la cobertura a nivel nacional (MINEDUC, 2006), como consecuencia de la norma constitucional del año 2003 que establece la obligatoriedad de 12 años de enseñanza, y al interés por continuar estudios superiores que se ha producido en los últimos 10 años, observándose una aceleración en la tasa de matrículas en este nivel educacional (Schmal, Ruiz, Donoso \& Schaffernicht, 2007). Se excluyeron a todos aquellos sujetos que presentaron alguna dificultad para comprender las instrucciones y/o que estuvieran físicamente discapacitados. Los datos de la distribución de la muestra se presentan en la Tabla 2.

\section{Instrumentos}

- Índice LSI-A

Se aplicó el Índice LSI-A de Neugarten y colaboradores (1961; 1996), el cual consta de 20 ítems (afirmaciones), que se responden con "De acuerdo"/ "En desacuerdo" / "??". Se puntúan 0 o 1 según sea la dirección de la respuesta. Si la 
Tabla 2. Características sociodemográficas de la muestra y distribución de frecuencias de los participantes del estudio ( $\mathrm{n}=473$ )

\begin{tabular}{|c|c|c|c|c|c|c|c|c|c|c|c|}
\hline \multirow[b]{2}{*}{ Edad } & \multirow{2}{*}{$\begin{array}{c}\text { Ingresos } \\
\text { N. Educ. } \\
\text { Sexo }\end{array}$} & \multicolumn{3}{|c|}{$0-\$ 84.000$} & \multicolumn{3}{|c|}{$\$ 85.000-\$ 264.000$} & \multicolumn{3}{|c|}{$\$ 265.000$ o más } & \multirow{2}{*}{$\begin{array}{l}\text { Total } \\
\text { sexo }\end{array}$} \\
\hline & & Básica & Media & Superior & Básica & Media & Superior & Básica & Media & Superior & \\
\hline \multirow{3}{*}{$\begin{array}{l}30-44 \\
\text { años }\end{array}$} & Masculino & 10 & 10 & 6 & 11 & 10 & 10 & 7 & 11 & 10 & 85 \\
\hline & Femenino & 9 & 10 & 11 & 11 & 9 & 10 & 5 & 9 & 10 & 84 \\
\hline & Total Edad & 19 & 20 & 17 & 22 & 19 & 20 & 12 & 20 & 20 & 169 \\
\hline \multirow{3}{*}{$\begin{array}{l}45-59 \\
\text { años }\end{array}$} & Masculino & 10 & 10 & 5 & 8 & 10 & 9 & 4 & 10 & 10 & 76 \\
\hline & Femenino & 9 & 10 & 7 & 9 & 10 & 10 & 3 & 10 & 10 & 78 \\
\hline & Total Edad & 19 & 20 & 12 & 17 & 20 & 19 & 7 & 20 & 20 & 154 \\
\hline \multirow{3}{*}{$\begin{array}{l}60-75 \\
\text { años }\end{array}$} & Masculino & 10 & 7 & 4 & 9 & 11 & 9 & 6 & 9 & 10 & 75 \\
\hline & Femenino & 9 & 8 & 6 & 9 & 7 & 10 & 9 & 8 & 9 & 75 \\
\hline & Total Edad & 19 & 15 & 10 & 18 & 18 & 19 & 15 & 17 & 19 & 150 \\
\hline \multicolumn{2}{|c|}{ Total N. Educacional } & 57 & 55 & 39 & 57 & 57 & 58 & 34 & 57 & 59 & 473 \\
\hline \multicolumn{2}{|c|}{ Total Ingreso } & & 151 & & & 172 & & & 150 & & 473 \\
\hline
\end{tabular}

respuesta era “?” el puntaje es “0”. Por tanto, el puntaje total que una persona puede obtener fluctúa entre 0 y 20 puntos (a mayor puntaje mayor satisfacción vital). A los participantes se les entregó las siguientes instrucciones:

Aqui hay algunas afirmaciones acerca de la vida en general. Lea cada afirmación de la lista, y si está de acuerdo ponga una X en el espacio que está debajo de la palabra "de acuerdo”. Si no está de acuerdo con la afirmación, ponga una $\boldsymbol{X}$ en el espacio que está debajo de la palabra "en desacuerdo”. Si no está seguro de ninguna de las alternativas anteriores, ponga una $\mathbf{X}$ debajo del espacio "?". Por favor, asegúrese de responder todas las preguntas.

\section{- Escala de Autoestima}

Se utilizó la validación chilena de la Escala de Autoestima de Rosenberg (1965), realizada por Rojas-Barahona, Zegers y Förster (2008). La prueba consta de 10 afirmaciones en torno a los sentimientos o pensamientos que tiene la persona sobre ella, 5 de ellas direccionados positivamente (ítems 1 , $2,4,6$ y 7 son positivos) y 5 negativamente (ítems $3,5,8$, 9 y 10 son negativos). La graduación de respuestas es de 4 puntos, desde 1 (totalmente en desacuerdo) a 4 (totalmente de acuerdo), por tanto, el puntaje mínimo son 10 y el máximo 40. Se les pide a los participantes marcar con una " $X$ " la respuesta que más lo identifica.

Un ejemplo de afirmación positiva es (ítem 2):

Creo que tengo un buen número de cualidades.

Un ejemplo de afirmación negativa es (ítem 10):

$A$ veces pienso que no soy bueno/a para nada.

\section{Procedimiento}

Las escalas LSI-A y LSI-B de Neugarten y colaboradores $(1961 ; 1996)$ fueron traducidas desde el inglés al español y evaluadas por 7 jueces expertos, tanto en idioma como en contenidos, considerando una concordancia mínima de 5 de ellos, equivalente al $71 \%$ de acuerdo. Las diferencias entre la versión original y la versión traducida fueron discutidas con el fin de mejorar la versión final, manteniendo el sentido original de las afirmaciones. Los criterios considerados en la validación de expertos fueron los siguientes: a) mantención del sentido e intencionalidad de las afirmaciones, b) lenguaje adecuado al contexto chileno, y c) revisión de aspectos formales del instrumento. Se realizó una primera aplicación piloto a 45 sujetos con características similares a los de la muestra definitiva, y se les pidió que eligieran entre las dos escalas, aquella que les resultara más fácil contestar. Unánimemente escogieron el LSI-A, motivo por el cual se optó por validar este instrumento. Posteriormente se realizó un segundo pilotaje donde se aplicó esta escala a 100 sujetos (también con características similares a la muestra definitiva) y a partir de estos resultados se hicieron los ajustes necesarios.

Para la fase de aplicación definitiva, se confeccionó un cuadernillo compuesto de cuatro partes: a) Presentación e Instrucciones para responder, b) Consentimiento Informado, c) Antecedentes personales: edad, sexo, nivel educacional y nivel de ingresos per cápita familiar, d) Escala de Satisfacción Vital (LSI-A) (Neugarten et al., 1961; 1996) y e) Escala de Autoestima de Rosenberg (1965), la versión validada en Chile por Rojas-Barahona, Zegers y Förster (2008). Fue aplicado por un grupo de encuestadores, todos alumnos de los últimos años de la Escuela de Psicología de la Universidad de los Andes, quienes fueron capacitados por el equipo de investigadores. La escala fue respondida individualmente, en un lugar silencioso, donde el participante estaba sentado durante toda la prueba.

\section{Análisis de datos}

Una vez obtenida la información, se determinaron las medias, desviaciones estándar, mínimos y máximos de la Escala LSI-A, para el total de participantes y para cada 
grupo de edad, sexo, nivel educacional y nivel de ingreso. Se realizó un análisis de los ítems considerando la media y la desviación estándar, asimetría y curtosis de cada uno, la correlación ítem-test, el coeficiente Alfa si el ítem era eliminado y la correlación de cada ítem con el puntaje total de la Escala de Autoestima de Rosenberg. Con el fin de determinar si existían diferencias en los resultados de las variables edad, nivel educacional y nivel de ingreso, se realizó un análisis de la varianza (ANOVA) y como test a posteriori Tukey b o Tamhane dependiendo del resultado de la prueba de Levene. Para determinar si había diferencias según sexo se utilizó Prueba $t$ para muestras independientes. El análisis de un posible sesgo de género en el instrumento se realizó a través de una tabla de contingencia de cada puntaje total de la Escala y la frecuencia de respuesta de hombres y mujeres, y se aplicó la prueba de $X^{2}$ para establecer la independencia entre el sexo y el puntaje total obtenido.

Para estimar la confiabilidad se calculó el coeficiente Alfa de Cronbach y se consideró que un valor superior a 0.75 indicaría que la consistencia interna del instrumento sería aceptable a buena, según los criterios de Henerson, Lyons y Taylor (1987), para escalas de autoreporte. Se realizó también un Análisis Factorial de Componentes Principales para determinar la validez de constructo del instrumento, utilizando como criterios los requisitos del test de Kaiser-Meyer-Olkin (KMO) y el test de esfericidad de Bartlett. Para determinar el número de factores se consideró que los autovalores fueran superiores a 1. Se utilizó el método de normalización Varimax y se analizaron los valores de los pesos factoriales rotados confirmando que su agrupación correspondiera a los factores teóricos del instrumento. La validez concurrente se estimó a través de un análisis de correlación de Pearson entre los puntajes del LSI-A y la Escala de Autoestima de Rosenberg.

\section{Resultados}

\section{Diferencias en la Satisfacción Vital según sexo, edad, nivel educacional y nivel de ingresos}

La media del Índice LSI-A para el total de la muestra fue de 14.36 puntos con una desviación estándar de 3.86. En la variable nivel educacional se observa un ascenso en las medias a medida que aumentan los años de estudio, y estas diferencias son significativas entre las medias de las categorías $\left(\mathrm{F}_{(2.221)}=17.069 ; p=0.000\right)$, siendo quienes han cursado sólo enseñanza básica los que presentan la media más baja (13.01), mientras que quienes tienen un nivel de escolaridad superior (técnica o universitaria) tienen la media más alta de satisfacción vital (14.51). Sin embargo, esta diferencia se encuentra entre quienes tienen enseñanza básica y los otros dos grupos $\left(\mathrm{F}_{(2,470)}=14.373 ; p=0.000\right)$. Las varianzas de los grupos son homogéneas $\left(\mathrm{F}_{(2,470)}=0.260\right.$; $p=0.771)$ y el rango más amplio de puntajes se encuentra en el nivel superior (1-20) (ver Tabla 3).

El resultado de la Satisfacción Vital para los niveles de ingreso de los participantes indica que también hay un aumento en la media a medida que el ingreso es mayor $\left(\mathrm{F}_{(2,470)}=14.309 ; p=0.000\right)$, donde la diferencia está entre el grupo de mayor ingreso y los otros dos (con valor $\mathrm{p}<$ 0.001 ); entre los dos ingresos más bajos no se observan diferencias $(p=0.505)$. El rango más amplio de puntajes se encuentra en el nivel bajo (1-20), aunque en todos los grupos se alcanza el máximo posible (ver Tabla 3 ).

No se observan diferencias significativas por edad $\left(\mathrm{F}_{2}\right.$ $\left.{ }_{2211}=0.124 ; p=0.884\right)$ ni por sexo $\left(\mathrm{F}_{(1,222)}=1.114 ; p=0.292\right)$, situación que concuerda con los análisis realizados por Neugarten y colaboradores $(1961 ; 1996)$; sin embargo, las medias de esta última variable son menores a las reportadas por los autores (17.9 en mujeres y 17.5 en hombres).

Tabla 3: Estadísticos descriptivos del puntaje total por cada grupo de edad, sexo, nivel educacional e ingresos

\begin{tabular}{|c|c|c|c|c|c|c|}
\hline Variable & Categoría & $\mathrm{N}$ & Media & D.S. & Mínimo & Máximo \\
\hline \multirow{3}{*}{ Edad } & $30-44$ & 169 & 13.75 & 3.791 & 2 & 20 \\
\hline & $45-59$ & 154 & 13.56 & 3.904 & 2 & 20 \\
\hline & $60-75$ & 150 & 13.85 & 4.114 & 1 & 20 \\
\hline \multirow{2}{*}{ Sexo } & Femenino & 237 & 13.58 & 3.901 & 2 & 20 \\
\hline & Masculino & 236 & 13.76 & 3.958 & 1 & 20 \\
\hline \multirow{3}{*}{ Nivel Educacional } & Básica & 148 & 13.01 & 3.846 & 2 & 20 \\
\hline & Media & 169 & 13.61 & 3.939 & 1 & 20 \\
\hline & Superior & 156 & 14.51 & 3.870 & 1 & 20 \\
\hline \multirow{3}{*}{ Ingreso (miles \$) } & $0-84$ & 151 & 12.79 & 4.326 & 1 & 20 \\
\hline & $85-264$ & 172 & 13.37 & 3.763 & 2 & 20 \\
\hline & 265 o más & 150 & 15.05 & 3.305 & 4 & 20 \\
\hline Total & & 473 & 14.36 & 3.86 & 1 & 20 \\
\hline
\end{tabular}


El análisis de $X^{2}$ que compara las frecuencias de hombres y mujeres en la distribución de puntajes totales de satisfacción vital, indica que no hay diferencias estadísticamente significativas en cada puntaje $\left(X_{(19)}=11.142 ; p=0.919\right)$, lo que implicaría que el LSI-A no presenta sesgo de género para la muestra estudiada. La distribución de frecuencias de los puntajes totales para hombres y mujeres se presenta en la Tabla 4.

En la Tabla 5 se muestran las medias, desviaciones estándar, asimetrías, curtosis, correlaciones ítem-test, alfa de Cronbach si se elimina el ítem y la correlación con el puntaje total de la Escala de Autoestima de Rosenberg de cada ítem. Cabe destacar que son los ítemes 5 y 20 los que presentan las medias más bajas $(0.31$ y 0.32 , respectivamente) y el ítem 8 con la media más alta y la menor desviación estándar de todos los ítems $(M=0.90, D E=0.302)$. En cuanto a la asimetría y la curtosis de los ítems, si bien presentan desviaciones respecto de una distribución normal de acuerdo con los valores de los errores típicos de ambas medidas de distribución, se consideran en un rango de normalidad (Asimetría $=0,112$; Curtosis $=0,224$ dado que es menor a 1.96 que es el límite de 1 desviación estándar en la curva normal). La correlación ítem-test es superior a 0.3 en la mayoría de los ítems, con excepción de los ítems 5, 11, 15, 16 , y 20 que tienen correlaciones que fluctúan entre 0.154 y 2.90. Mención aparte merece el ítem 8 que presenta una correlación negativa con el test y es el único que no presenta una correlación con la Escala de Autoestima de Rosenberg (1965); todos los otros ítems presentan correlaciones significativas con dicha escala.

\section{Estimación de la Confiabilidad}

La confiabilidad del instrumento se realizó a través de un análisis de multipartición donde se obtuvo un coeficiente Alfa de Cronbach de 0.79, lo que demuestra una buena consistencia interna del instrumento (Henerson, Lyons y Taylor, 1987). Sin embargo, como se mencionó anteriormente, la correlación ítem-test para la afirmación 8 es inversa (-0.115), razón por la cual se recomienda excluirlo del análisis de confiabilidad (Hair, Tatham, Black y Anderson, 1999). El coeficiente Alfa de Cronbach luego de eliminar el ítem 8, es de 0.80. El índice de confiabilidad es considerado bueno para cada grupo estudiado (edad, sexo o nivel educacional). En la estratificación por ingreso per cápita los valores fluctúan entre 0.74 y 0.83 , considerados aceptables a buenos (Tabla 6).

Tabla 4: Distribución de frecuencia de puntajes totales obtenido por hombres y mujeres

\begin{tabular}{|c|c|c|c|}
\hline Puntaje Total & Frecuencia Hombres & Frecuencia Mujeres & Frecuencia Total \\
\hline 1 & 1 & 0 & 1 \\
\hline 2 & 3 & 2 & 5 \\
\hline 3 & 3 & 3 & 6 \\
\hline 4 & 1 & 3 & 4 \\
\hline 5 & 2 & 3 & 5 \\
\hline 6 & 3 & 2 & 5 \\
\hline 7 & 4 & 5 & 9 \\
\hline 8 & 9 & 4 & 13 \\
\hline 9 & 7 & 10 & 17 \\
\hline 10 & 11 & 11 & 22 \\
\hline 11 & 12 & 22 & 34 \\
\hline 12 & 20 & 19 & 39 \\
\hline 13 & 17 & 19 & 36 \\
\hline 14 & 27 & 23 & 50 \\
\hline 15 & 27 & 20 & 47 \\
\hline 16 & 27 & 24 & 51 \\
\hline 17 & 22 & 26 & 48 \\
\hline 18 & 22 & 27 & 49 \\
\hline 19 & 12 & 9 & 21 \\
\hline 20 & 6 & 5 & 11 \\
\hline Total & 236 & 237 & 473 \\
\hline
\end{tabular}


Tabla 5. Estadísticos descriptivos de los ítems del LSI-A

\begin{tabular}{|c|c|c|c|c|c|c|c|}
\hline & Media & D.S. & Asimetría & Curtosis & $\begin{array}{l}\text { Correlación } \\
\text { item-test }(*)\end{array}$ & $\begin{array}{l}\text { Alfa si se } \\
\text { elimina el } \\
\text { ítem }\end{array}$ & $\begin{array}{c}\text { Correlación } \\
\text { con EAR }\end{array}$ \\
\hline $\begin{array}{l}\text { 1. A medida que pasan los años, las cosas parecen mejores } \\
\text { de lo que yo pensé que serían. }\end{array}$ & ,71 & ,453 &,- 942 & $-1,118$ & ,367 &, 780 &, $197(* *)$ \\
\hline $\begin{array}{l}\text { 2. He tenido más oportunidades en la vida que la mayoría } \\
\text { de las personas que conozco. }\end{array}$ & ,62 & ,487 &,- 475 & $-1,782$ &, 370 & ,779 &, $218(* *)$ \\
\hline 3. Esta es la etapa más monótona de mi vida. & ,71 & ,454 &,- 930 & $-1,139$ & ,371 & ,779 &, $307(* *)$ \\
\hline 4. Soy tan feliz como cuando era más joven. & ,68 & ,465 &,- 799 & $-1,367$ & ,473 & ,773 &, $215(* *)$ \\
\hline 5. Mi vida podría ser más feliz de lo que es ahora. &, 31 & ,464 &, 810 & $-1,350$ & ,289 & ,785 &, $208(* *)$ \\
\hline 6. Estos son los mejores años de mi vida. &, 56 & ,497 &,- 252 & $-1,945$ &, 418 & ,776 &, $093(*)$ \\
\hline $\begin{array}{l}\text { 7. La mayoría de las cosas que hago son aburridas o } \\
\text { monótonas }\end{array}$ &, 77 & ,424 & $-1,256$ &,- 424 & ,427 &, 776 &, $299(* *)$ \\
\hline $\begin{array}{l}\text { 8. Espero que en el futuro me ocurran algunas cosas inte- } \\
\text { resantes y placenteras }\end{array}$ & ,90 & ,302 & $-2,648$ & 5,033 &,- 115 &, 801 &,- 067 \\
\hline $\begin{array}{l}\text { 9. Las cosas que hago son tan interesantes para mí como } \\
\text { lo eran antes. }\end{array}$ &, 80 & ,399 & $-1,515$ & ,296 &, 454 & ,775 &, $251(* *)$ \\
\hline 10. Me siento viejo y un tanto cansado. & ,71 & ,452 &,- 953 & $-1,096$ & ,431 & ,776 &, $283(* *)$ \\
\hline 11. Siento mis años, pero no es algo que me moleste. &, 74 & 439 & $-1,098$ &,- 799 &, 154 & ,793 &, $117(*)$ \\
\hline $\begin{array}{l}\text { 12. Al mirar mi vida hacia atrás, me siento bastante } \\
\text { satisfecho }\end{array}$ &, 82 & ,384 & $-1,674$ & ,805 &, 518 & ,772 &, $251(* *)$ \\
\hline 13. No cambiaría mi vida pasada aunque pudiera. & ,63 & ,484 &,- 522 & $-1,735$ & ,335 & ,782 &, $139(* *)$ \\
\hline $\begin{array}{l}\text { 14. Comparado con otras personas de mi edad, he tomado } \\
\text { muchas decisiones tontas en mi vida. }\end{array}$ & ,66 & ,476 &,- 656 & $-1,576$ & ,398 & ,778 &, $246(* *)$ \\
\hline $\begin{array}{l}\text { 15. Comparado con otras personas de mi edad, tengo una } \\
\text { buena apariencia. }\end{array}$ &, 83 & ,375 & $-1,771$ & 1,141 & ,268 & ,785 &, $185(* *)$ \\
\hline $\begin{array}{l}\text { 16. He hecho planes para hacer cosas dentro de este mes } \\
\text { o este año. }\end{array}$ &, 85 & ,353 & $-2,013$ & 2,060 &, 177 &, 790 &, $116(*)$ \\
\hline $\begin{array}{l}\text { 17. Cuando pienso en mi vida pasada, me doy cuenta que } \\
\text { no conseguí la mayor parte de las cosas importantes } \\
\text { que quise. }\end{array}$ &, 57 & ,495 &,- 287 & $-1,926$ & ,320 & ,783 &, $221(* *)$ \\
\hline $\begin{array}{l}\text { 18. Comparado con otras personas, me siento abatido de- } \\
\text { masiado frecuentemente }\end{array}$ & ,78 &, 416 & $-1,342$ &,- 199 & ,487 & ,773 &, $315(* *)$ \\
\hline 19. He conseguido gran parte de lo que esperé de mi vida &, 75 & ,436 & $-1,136$ &,- 713 &, 518 & ,770 &, $253(* *)$ \\
\hline $\begin{array}{l}\text { 20. A pesar de lo que la gente dice, la mayoría de las personas } \\
\text { están poniéndose peores, no mejores }\end{array}$ & ,32 & ,466 & ,788 & $-1,384$ & ,290 & ,785 &, $169(* *)$ \\
\hline
\end{tabular}

(*) La correlación ítem-test fue calculada considerando la escala completa

Tabla 6. Confiabilidad del test en cada grupo de estudio utilizando Alfa de Cronbach

\begin{tabular}{|c|c|c|}
\hline & & Alpha de Cronbach \\
\hline \multirow{2}{*}{ Sexo } & Hombre & 0.80 \\
\hline & Mujer & 0.80 \\
\hline \multirow{3}{*}{ Nivel Educacional } & Básica & 0.77 \\
\hline & Media & 0.81 \\
\hline & Superior & 0.81 \\
\hline \multirow{3}{*}{ Ingresos (Miles \$) } & $0-84$ & 0.83 \\
\hline & $85-264$ & 0.77 \\
\hline & 265 o más & 0.74 \\
\hline \multirow{3}{*}{ Edad (años) } & $30-44$ & 0.78 \\
\hline & $45-59$ & 0.80 \\
\hline & $60-75$ & 0.83 \\
\hline
\end{tabular}

\section{Validez de constructo}

Luego de confirmar los supuestos requeridos para un Análisis Factorial Exploratorio (AFE) $(\mathrm{KMO}=0.841 \mathrm{y}$ Test de Bartlett $\left.X^{2}{ }_{(190)}=1646.64 ; p=0.000\right)$, se realizó un Análisis Factorial de Componentes Principales con las respuestas a los 20 ítemes, y luego un nuevo análisis excluyendo el ítem 8 , dada la escasa consistencia interna que éste presentó. En ambos casos se observa que los ítemes se agrupan en 5 factores utilizando el criterio de Kaiser (autovalores mayores que 1) que explican, en el primer caso, 
un $48.69 \%$ de la varianza total de los puntajes obtenidos por los sujetos, y en el segundo caso un $50.62 \%$. El detalle de los porcentajes de varianza explicados en cada factor para ambos análisis se presenta en la Tabla 7, donde cabe destacar que el porcentaje de varianza explicada aumenta para todos los factores al eliminar el ítem 8 .

La matriz de componentes rotados (método de normalización Varimax) muestra los pesos factoriales de cada ítem en el componente al que se asocia. En la tabla 8 se presentan los 5 factores con sus respectivos ítems y los pesos factoriales para ambos análisis (20 ítems y excluyendo el ítem 8 ), donde se observa que la conformación de los mismos no varía y los pesos de cada ítem son similares. El Factor 1 quedó constituido por los ítemes 1, 2, 4, 6, 12, 13 y 19 y sus contenidos permiten nominarlo Congruencias entre logros deseados y obtenidos; el Factor 2 incluye los ítems 3, 7, 10 y 18 y se lo denominó Entusiasmo; el Factor 3 agrupa los ítems 5, 14 y 17 y 20 y se lo llamó Tono Emocional; el Factor 4 consta de los ítems 9, 11 y 15, representando el Autoconcepto; y el Factor 5 incluye los ítem 8 y 16 y corresponde a Resolución y Fortaleza. La agrupación de los ítemes en los factores que constituyen el LSI-A se realizó considerando el peso factorial y el contenido del ítem. Es así que si bien para el ítem 9 se obtuvo un peso factorial de 0.439 (Factor 2) y de 0.434 (Factor 4), atendiendo a su contenido se optó por dejarlo en el Factor 4. Respecto del ítem 20 se planteó una decisión similar, con un peso factorial de 0.384 (Factor 1) y 0.343 (Factor 3 ) y se lo dejó, por su contenido, en el factor 3. Las confiabilidades de los dos primeros factores son consideradas aceptables para este tipo de escalas, más aun cuando el número de ítems es reducido. Los factores 3, 4 y 5 presentaron un bajo coeficiente de Alfa de Cronbach (menor a 0.52), lo que indica que estos factores tienen una escasa consistencia interna y que la proporción de varianza que comparten es baja, lo cual es esperable dado el número de ítems que conforman dichos factores (Nunnally \& Bernstein, 1995).

\section{Validez Concurrente}

Para analizar la validez concurrente se realizó un análisis de Correlación de Pearson entre la LSI-A y otro instrumento o criterio establecido previamente, que tuviera una relación teórica y que fuese medido al mismo tiempo (Aiken, 1985; Croker \& Algina, 1986), en este caso la Escala de Autoestima de Rosenberg (1965). La correlación entre ambas fue directa $(\mathrm{r}=0.455)$ y significativa $(\mathrm{p}<0.01)$, lo que indica que ambas escalas medirían la autopercepción de las personas en la misma dirección.

\section{Discusión y Conclusiones}

El tamaño muestral de este estudio y su estratificación por edad, sexo, nivel educacional y nivel de ingreso percápita del grupo familiar, junto a unidades de análisis homogéneas al interior de los diversos estratos y entre ellos, constituye el fundamento de la precisión de las estimaciones globales obtenidas. No se apreciaron sesgos en los puntajes totales de satisfacción vital para el sexo, sí diferencias significativas para las medias de puntajes según escolaridad, siendo más bajas entre quienes tienen menor escolaridad y los otros dos grupos. La media de puntajes también baja a medida que disminuye el nivel de ingreso, siendo las diferencias significativas para este grupo comparado con los otros dos. De lo anterior se puede concluir que la satisfacción vital de los sujetos estudiados no se ve afectada por las variables sexo y edad, sí por el nivel de escolaridad e ingreso. Se destaca que no se den variaciones significativas respecto de la edad, ya que este comportamiento muestral ratifica la hipótesis de que el LSI-A puede ser empleado

Tabla 7. Porcentaje de la varianza total explicada

\begin{tabular}{|c|c|c|c|c|c|c|c|c|c|c|}
\hline & & \multicolumn{3}{|c|}{ Autovalores Iniciales } & \multicolumn{4}{|c|}{ Solución no rotada inicial } & \multicolumn{2}{|c|}{$\begin{array}{c}\text { Solución con rotación } \\
\text { Varimax }\end{array}$} \\
\hline & Factores & Total & $\begin{array}{l}\text { Porcentaje } \\
\text { Varianza } \\
\text { Explicada } \\
\end{array}$ & $\begin{array}{c}\text { Porcentaje } \\
\text { Varianza } \\
\text { Acumulada }\end{array}$ & Total & $\begin{array}{l}\text { Porcentaje } \\
\text { Varianza } \\
\text { Explicada } \\
\end{array}$ & $\begin{array}{l}\text { Porcentaje } \\
\text { Varianza } \\
\text { Acumulada } \\
\end{array}$ & Total & $\begin{array}{c}\text { Porcentaje } \\
\text { Varianza } \\
\text { Explicada } \\
\end{array}$ & $\begin{array}{l}\text { Porcentaje } \\
\text { Varianza } \\
\text { Acumulada }\end{array}$ \\
\hline \multirow[t]{5}{*}{20 ítemes } & 1 & 4.393 & 21.963 & 21.963 & 4.393 & 21.963 & 21.963 & 2.740 & 13.700 & 13.700 \\
\hline & 2 & 1.705 & 8.523 & 30.486 & 1.705 & 8.523 & 30.486 & 2.605 & 13.024 & 26.724 \\
\hline & 3 & 1.304 & 6.518 & 37.004 & 1.304 & 6.518 & 37.004 & 1.741 & 8.703 & 35.427 \\
\hline & 4 & 1.192 & 5.958 & 42.962 & 1.192 & 5.958 & 42.962 & 1.391 & 6.953 & 42.380 \\
\hline & 5 & 1.146 & 5.729 & 48.692 & 1.146 & 5.729 & 48.692 & 1.262 & 6.311 & 48.692 \\
\hline \multirow[t]{5}{*}{19 ítemes } & 1 & 4.374 & 23.020 & 23.020 & 4.374 & 23.020 & 23.020 & 2.711 & 14.270 & 14.270 \\
\hline & 2 & 1.676 & 8.819 & 31.839 & 1.676 & 8.819 & 31.839 & 2.563 & 13.487 & 27.757 \\
\hline & 3 & 1.266 & 6.662 & 38.501 & 1.266 & 6.662 & 38.501 & 1.745 & 9.182 & 36.938 \\
\hline & 4 & 1.169 & 6.151 & 44.651 & 1.169 & 6.151 & 44.651 & 1.368 & 7.197 & 44.136 \\
\hline & 5 & 1.133 & 5.964 & 50.615 & 1.133 & 5.964 & 50.615 & 1.231 & 6.479 & 50.615 \\
\hline
\end{tabular}


Tabla 8. Resumen de la organización factorial del LSI-A y los pesos factoriales rotados, el autovalor y alfa de Cronbach de cada factor para 20 y 19 ítems

\begin{tabular}{|c|c|c|c|c|c|c|}
\hline \multirow[t]{2}{*}{ Factor } & & \multicolumn{2}{|l|}{20 ítems } & \multicolumn{3}{|l|}{19 ítems } \\
\hline & & $\begin{array}{l}\text { Peso } \\
\text { factorial }\end{array}$ & Autovalor & $\begin{array}{l}\text { Peso } \\
\text { factorial }\end{array}$ & Autovalor & $\begin{array}{l}\text { Alfa de } \\
\text { Cronbach }\end{array}$ \\
\hline \multirow[t]{7}{*}{ Factor 1} & $\begin{array}{l}\text { 1. A medida que pasan los años, las cosas parecen mejores de } \\
\text { lo que yo pensé que serían. }\end{array}$ & .589 & \multirow{7}{*}{4.393} & .598 & \multirow{7}{*}{4.374} & \multirow{7}{*}{.734} \\
\hline & $\begin{array}{l}\text { 2. He tenido más oportunidades en la vida que la mayoría de } \\
\text { las personas que conozco. }\end{array}$ & .521 & & .518 & & \\
\hline & 4. Soy tan feliz como cuando era más joven. & .572 & & .571 & & \\
\hline & 6. Estos son los mejores años de mi vida. & .654 & & ,646 & & \\
\hline & 12. Al mirar mi vida hacia atrás me siento bastante satisfecho & .670 & & 669 & & \\
\hline & 13. No cambiaría mi vida pasada aunque pudiera. & .425 & & .417 & & \\
\hline & 19. He conseguido gran parte de lo que esperé de mi vida & 689 & & .687 & & \\
\hline \multirow[t]{4}{*}{ Factor 2} & 3. Esta es la etapa más monótona de mi vida. & .730 & \multirow{4}{*}{1.705} & .731 & \multirow{4}{*}{1.676} & \multirow{4}{*}{.706} \\
\hline & $\begin{array}{l}\text { 7. La mayoría de las cosas que hago son aburridas o } \\
\text { monótonas }\end{array}$ & .718 & & .715 & & \\
\hline & 10. Me siento viejo y un tanto cansado. & .685 & & 691 & & \\
\hline & $\begin{array}{l}\text { 18. Comparado con otras personas, me siento abatido dema- } \\
\text { siado frecuentemente }\end{array}$ & .526 & & .513 & & \\
\hline \multirow[t]{4}{*}{ Factor 3} & 5. Mi vida podría ser más feliz de lo que es ahora. & .343 & \multirow{4}{*}{1.304} & .412 & \multirow{4}{*}{1.266} & \multirow{4}{*}{.521} \\
\hline & $\begin{array}{l}\text { 14. Comparado con otras personas de mi edad he tomado } \\
\text { muchas decisiones tontas en mi vida. }\end{array}$ & .716 & & .723 & & \\
\hline & $\begin{array}{l}\text { 17. Cuando pienso en mi vida pasada, me doy cuenta que no } \\
\text { conseguí la mayor parte de las cosas importantes que quise. }\end{array}$ & .672 & & .664 & & \\
\hline & $\begin{array}{l}\text { 20. A pesar de lo que la gente dice, la mayoría de las personas } \\
\text { están poniéndose peores, no mejores }\end{array}$ & .331 & & .343 & & \\
\hline \multirow[t]{3}{*}{ Factor 4} & $\begin{array}{l}\text { 9. Las cosas que hago son tan interesantes para mí como lo } \\
\text { eran antes. }\end{array}$ & .434 & \multirow{3}{*}{1.192} & .434 & \multirow{3}{*}{1.169} & \multirow{3}{*}{.354} \\
\hline & 11. Siento mis años pero no es algo que me moleste. & .592 & & .611 & & \\
\hline & $\begin{array}{l}\text { 15. Comparado con otras personas de mi edad tengo una buena } \\
\text { apariencia. }\end{array}$ & .548 & & .490 & & \\
\hline \multirow[t]{2}{*}{ Factor 5} & $\begin{array}{l}\text { 8. Espero que en el futuro me ocurran algunas cosas interesantes } \\
\text { y placenteras. }\end{array}$ & .540 & \multirow[b]{2}{*}{1.146} & --- & \multirow[b]{2}{*}{1.133} & \multirow[b]{2}{*}{$.111^{*}$} \\
\hline & $\begin{array}{l}\text { 16. He hecho planes para hacer cosas dentro de este mes o } \\
\text { este año. }\end{array}$ & .705 & & .805 & & \\
\hline
\end{tabular}

*La confiabilidad de este factor incluye ambos ítemes.

para medir la satisfacción vital y el bienestar subjetivo en cualquier edad en la adultez.

Las medias de puntajes fluctuaron entre 12.79 y 15.05 y D.S entre 3.305 y 4.326 , con un mínimo de 0 puntos y un máximo de 20 , comportándose la muestra de acuerdo a los puntajes teóricos posibles. La distribución de los puntajes del LSI-A en esta aplicación se aproxima a una curva normal con una ligera asimetría negativa. No se realizaron estudios de corte para diferenciar entre sujetos con alta y baja satisfacción vital, asunto que no formó parte del diseño de investigación.

La consistencia interna del instrumento, en independencia de variables sociodemográficas, fue de 0.79 cuando se incluyó el ítem 8 y mejoró a 0.80 cuando éste se eliminó, medida considerada buena (Henerson, Lyons \& Taylor, 1987). Aunque el LSI-A se desarrolló originalmente como una medida del bienestar psicológico entre AM, este estudio muestra que el instrumento tiene una buena consistencia interna cuando se usa en otros grupos de edad. Se obtuvo un alfa de Cronbach que fluctuó entre 0.78 para el grupo de 30-44 años y 0.83 para el grupo de 60 a 75 años, ambos valores considerados buenos. Con todo, su confiabilidad es mejor para el grupo de mayor edad. No se apreciaron variaciones en la consistencia interna para la variable sexo (0.80); sí para la escolaridad (0.77 a 0.81$)$ y para el nivel de ingreso, donde las variaciones fueron mayores $(0.74 \mathrm{a}$ 0.83). Cabe destacar que la consistencia interna mejora a menor nivel de ingreso, hallazgo que puede interpretarse debido a que este grupo, posiblemente, estuvo menos preocupado por el manejo de impresiones, elemento que ha sido reportado por Park, Upshaw y Koh (1988), aunque se espera que ésta sea mayor en entrevistas cara a cara que cuando el instrumento se aplica anónimamente, como fue el caso de este estudio. 
El análisis del comportamiento individual de los ítemes se realizó para el total de la muestra; sin embargo, la decisión de eliminar el ítem 8 "Espero que en el futuro me ocurran cosas interesantes y placenteras" ("I expected some interesting and pleasent things to happen to me in the future") se justifica por su correlación inversa con el puntaje total del LSI-A. Además, esta decisión se adoptó luego de considerar el aumento del coeficiente Alpha, una vez eliminado el ítem y la correlación nula del ítem con el puntaje total de la Escala de Autoestima de Rosenberg (1965). Este comportamiento puede deberse al hecho de que es el único ítem que considera de forma explícita la dimensión de futuro en su redacción. Todos los demás, exceptuando el ítem 16, apuntan directa o indirectamente a una evaluación del bienestar subjetivo actual en comparación con el pasado, como fue la intención original de Neugarten y colaboradores $(1961 ; 1996)$.

El análisis de contenido, unido a sus correlaciones, permitió nominar a estos cinco factores con los mismos nombres que conformaron las dimensiones originales por Neugarten y colaboradores $(1961 ; 1996)$. El factor Congruencia entre objetivos deseados y alcanzados se encuentra representado por 7 ítemes (Factor 1); Entusiasmo por 4 ítemes (Factor 2), Tono emocional también por 4 ítemes (Factor 3); Autoconcepto por 3 ítemes (Factor 4) y Resolución y Fortaleza por 2 ítems (Factor 5), siendo una de ellas el número 8 . Se desprende de lo anterior que el LSI-A es una medida que evalúa con mayor precisión las dos primeras dimensiones (la tercera con algunos reparos de confiabilidad). Se recomienda que quienes quieran evaluar el autoconcepto recurran a otras escalas como por ejemplo, la escala de Autoestima de Rosenberg (1965; Rosenberg, Schooler, Schoenbach \& Rosenberg, 1995), que por su facilidad y simplicidad de aplicación se ha aplicado en una diversidad de países (por ejemplo, ver Cheng \& Page, 1989; Helbing, 1982; Okada \& Nagai, 1990; Rojas-Barahona, Zegers y Förster, 2008), mostrando por lo general ser válida y tener una alta confiabilidad. Respecto de la dimensión Resolución y Fortaleza se sugiere que la construcción de futuras escalas de satisfacción vital, y que se basen en el marco teórico de Neugarten y colaboradores $(1961 ; 1996)$, contemplen la inclusión de nuevos ítemes que apunten a esta dimensión.

En síntesis, la validez de constructo del LSI-A fue estudiada a partir del análisis factorial de componentes principales, confirmándose una organización factorial de cinco factores como inicialmente plantearon sus autores (Neugarten et al., 1961; 1996), siendo congruente con el cuerpo teórico presentado. Esta organización explica el $50.615 \%$ de la varianza total de los puntajes de los sujetos cuando se excluye el ítem 8 . Dado que los factores corresponden a constructos psicológicos, parte de la variabilidad total también es explicada por otras variables que no están siendo medidas por el instrumento. Así por ejemplo, Diener y colaboradores (1999), al revisar los avances realizados en la medición del bienestar subjetivo en las últimas tres décadas, afirman que sería muy deseable que los investigadores incluyeran otras medidas complementarias que no fueran de autoreporte (índices biológicos, expresiones faciales, tiempo de reacción, entre otras), y que por ende, fortalecen la evaluación. Si bien la muestra se estratificó según edad, sexo, nivel de escolaridad y nivel de ingreso, no se consideró la variable salud, factor que Conrad-Glass y Jolly (1997) y Dorin (2007) destacan como variable que incide en la satisfacción vital y en el bienestar subjetivo, siendo ésta, una limitación del estudio no haberla incluido.

Otra de las limitaciones del LSI-A que se presenta es que no se estimó la validez predictiva, por lo tanto se desconoce su estabilidad en el tiempo en Chile, aspecto que no formó parte del diseño de investigación. Lobello, Underhill y Fine (2004) se refieren a la inestabilidad de la medida y se concuerda con ellos en que esta inestabilidad probablemente refleja la relativa variabilidad de la satisfacción vital a lo largo de la vida, lo que se fundamenta en las características propias de la vida humana, cuya trama está formada por una gama compleja de experiencias y oportunidades que varían de una época a otra y entre distintos individuos.

Dado que son constructor relacionados, para evaluar la validez concurrente del LSI-A se consideró la Escala de Autoestima de Rosenberg (1965), específicamente se utilizó la prueba validada en Chile por Rojas-Barahona y colaboradores (2008), obteniéndose una correlación directa $(\mathrm{r}=0.455)$ y significativa $(\mathrm{p}<0.01)$, lo que indica que ambas escalas medirían la autopercepción de las personas en la misma dirección.

Los resultados permiten concluir que el LSI-A es una medida confiable y válida desde el punto de vista de su contenido y estructura factorial para evaluar el constructo psicológico de satisfacción vital y la calidad de vida en adultos chilenos. Es especialmente útil cuando se quiere conocer la congruencia entre objetivos deseados y alcanzados, entusiasmo y tono emocional.

Los cinco factores obtenidos en el análisis factorial dan cuenta de $50.62 \%$ de la varianza total de los puntajes de los sujetos y la consistencia interna de la escala total es de 0.80 , valor que es considerado bueno para escalas que miden apreciación personal de un concepto.

El instrumento se sugiere conformarlo por 19 ítemes, eliminando el ítem 8 de la versión original. Se responde de acuerdo o en desacuerdo frente a cada afirmación. Se otorga un punto si se responde de acuerdo a los ítemes 1, 2, 4, 6, $9,11,12,13,15,16$, y 19 y en desacuerdo a los ítemes 3 , $5,7,10,14,17,18$ y 20 . A mayor puntaje, mayor grado de bienestar subjetivo y satisfacción vital.

La prueba no muestra sesgo según edad, pero sí diferencias en la media de puntajes según nivel de escolaridad y nivel socioeconómico, siendo el nivel de ingreso más alto donde se obtuvo la menor confiabilidad (0.74). Para 
todos los otros criterios de estratificación, la confiabilidad es aceptable a buena.

\section{Referencias}

Aiken, L. (1985). Three coefficients for analizing the reliability and validity of ratings. Educational and Psychological Measurement, 45, 121-140.

CASEN (2003). Módulos de Ingresos: Cuadros Ingresos 2003. Recuperado el 14 de mayo de 2007, de http://www.mideplan.cl/casen/ modulo_ingresos.html

Cheng, H. P. y Page, R. C. (1989). The relationships among sex, academic performance, anxiety, and self-esteem of junior high school students in Taiwan. Journal of Multicultural Couseling and Development, 17, 123-133.

Chiang, K., Lu, R., Chu, H., Chang, Y. \& Chou, K. (2008). Evaluation of the effect of a life review group program on self-esteem and life satisfaction in the elderly. International Journal of Geriatric Psychiatry, 23, 7-10.

Conrad-Glass, J. \& Jolly, G. R. (1997). Satisfaction in later life among women 60 or over. Educational Gerontology, 23, 297-314.

Croker, L. \& Algina, J. (1986). Introduction to classical and modern test theory. Forth Worth, FL: Harcourt Brace Jovanovich.

Diener, E., Suh, E. M., Lucas, R. E. \& Smith, H. L. (1999). Subjective well-bein: Three decades of progress. Psychological Bulletin, 125, 276-302.

Dorin, M. (2007). Online education of older adults and its relation to life satisfaction. Educational Gerontology, 33, 127-143.

Hair, J., Tatham, R., Black, W. \& Anderson, R. (1999). Análisis multivariante de datos. Madrid: Prentice Hall-Pearson.

Helbing, J. C. (1982). Zelfwaardering: Meeting en validiteit. Nederlands Tijdschrift Loor DePsychologie En Haar Grensgebieden, 37, $257-$ 277.

Henerson, M.E., Lyons, L. \& Taylor, C. (1987). How to measure attitudes. University of California. Los Ángeles. Sage Publications.

Inga, J. \& Vara, A. (2006). Factores asociados a la satisfacción de vida de adultos mayores de 60 años en Lima-Perú. Universitas Psychologica, $5,475-495$.

Levinson. D. (1978). The seasons of man's life. New York: Alfred Knopf.

Levinson. D \& Levinson. J. (1996). The seasons of a woman's life. New York: Alfred Knopf.
Lobello, S. G., Underhill, A. T. \& Fine, P. R. (2004). The reliability and validity of Life Satisfaction Index-A with survivors of traumatic brain injury. Brain Injury, 18, 1127-1134.

MINEDUC (2006). Anuario estadístico 2006 (Provisorio). Recuperado el 28 de agosto de 2008 de http://www.mineduc.cl/index2.php?id_ seccion $=1275 \&$ id_portal $=1 \&$ id_contenido $=5358$

Neugarten, B. L., Havighurst, R. J. \& Tobin, S. S. (1961). The measurement of Life Satisfaction. Journal of Gerontology, 16 134-143.

Neugarten, B. L., Havighurst, R. J. \& Tobin, S. S. (1996). The measurement of Life Satisfaction. D. A. Neugarten (Ed.) The meanings of age. Selected papers of Bernice L. Neugarten (pp. 296-323). Chicago: The University of Chicago Press.

Nunnally, J.C y Bernstein, I.J. (1995). Teoría de la Psicometría. New York: MacGraw Hill.

Okada, T. y Nagai, T. (1990). Self-esteem and anthrophobic-tendency in adolescent. Japanese Journal of Psychology, 60, 386-389.

Olson-Maddem J. H. (2008). Correltes and predictors of life satisfaction among 18 to 35-year olds: An exploration of the 'quarterlife crisis' phenomenon. Dissertation Abstracts International Section A: Humanities and Social Sciences, 68(8-A), p. 3298.

Park, K. B., Upshaw, H. S. \& Koh, S. D. (1988). East Asians' responses to western health items. Journal of Cross-Cultural Psychology, 19, 51-64.

Rojas-Barahona, C. A., Zegers, B. \& Förster, C. E. (2008). La Escala de Autoestima de Rosenberg: Validación para Chile en una muestra de jóvenes adultos, adultos y adultos mayores. Enviado a Publicar.

Rosenberg, M. (1965). Society and the adolescent self-image. Princeton: Princeton University Press.

Rosenberg, M., Schooler, C., Schoenbach, C. \& Rosenberg, F. (1995). Global self-esteem and specific self-esteem. American Sociological Review, 60, 141-156.

Schmal, R., Ruiz, R., Donoso, S. \& Schaffernicht, E. M. (2007). Factores que inciden en el financiamiento de los estudios Universitarios en Chile. Sociologias, 9, 316-339.

Sener, A., Terzioglu, R. G. \& Karabulut, E. (2007). Life satisfaction and leisure activities Turing men's retirement: A Turkish sample. Aging \& Mental Health, 11, 30-36.

Smith, J., Brochelt, M., Maier, H. \& Jopp, D. (2002). Health and well-being in the young old and oldest old. Journal of Social, 58, 715-73.

Staudinger, U. M., Fleeson, W. \& Baltes, P. B. (1999). Predictors of subjective physical health and global well being: Similarities and differences between the United States and Germany. Journal of Personality and Social Psychology, 76, 305-319. 